\title{
Reconstruction Parameters for ${ }^{111}$ In-Pentetreotide SPECT: Variability with Respect to Body Weight and Body Region
}

\author{
Arianna Massaro ${ }^{1}$, Silvia Cittadin ${ }^{1}$, Francesca Rossi ${ }^{2}$, Lucia Rampin ${ }^{1}$, Elena Banti ${ }^{1}$, Otello Nibale ${ }^{3}$, and \\ Domenico Rubello ${ }^{1}$ \\ ${ }^{\text {I}}$ PET Unit, Nuclear Medicine Service, S. Maria della Misericordia Hospital, Rovigo, Italy; ${ }^{2}$ Technician and Nursing Service, S. Maria \\ della Misericordia Hospital, Rovigo, Italy; and ${ }^{3}$ Medical Physic Service, S. Maria della Misericordia Hospital, Rovigo, Italy
}

This study, which was based on a large series of consecutive patients imaged by ${ }^{111} \mathrm{In}$-pentetreotide SPECT for a neuroendocrine tumor, evaluated variability in reconstruction parameters in relation to patient body weight and the body region imaged, looking for the possibility of standardizing such parameters. Methods: One hundred twenty-four patients underwent ${ }^{111} \mathrm{In}$ pentetreotide scintigraphy: 4- and 24-h whole-body and planar scans and a 24-h SPECT examination. All patients were injected with $140-150 \mathrm{MBq}$ of ${ }^{111}$ In-pentetreotide at least $1 \mathrm{wk}$ after somatostatin analogs had been discontinued. SPECT images were systematically acquired at the levels of the head, chest, and abdomen. SPECT was performed using a dual-head $\gamma$-camera with medium-energy collimators, step-and-shoot method, no circular orbit, a $64 \times 64$ matrix, and $30 \mathrm{~s}$ per view for a total of 64 views. Two reconstruction procedures were compared: the iterative method using 10 iterations and the filtered backprojection method using a Butterworth filter with different cutoffs and orders. Results: Optimal SPECT images were obtained by applying the Butterworth filter. The reconstruction parameters could be standardized for the head and chest but were more variable for the abdomen, mainly because ${ }^{111}$ In-pentetreotide is physiologically trapped in different intestinal areas and varies over time, especially in the liver, spleen, bowel, and urinary tract. Conclusion: Filtered backprojection using a Butterworth filter appears adequate for standardizing the reconstruction parameters for ${ }^{111}$ In-pentetreotide SPECT of the head and chest. Processing of abdominal images is more operator-dependent. A $150-\mathrm{MBq}$ dose of ${ }^{111}$ In-pentetreotide is recommended when planning multiple SPECT acquisitions in the same patient.

Key Words: ${ }^{111}$ In-pentetreotide; SPECT; reconstruction parameters; standardization; neuroendocrine tumors

J Nucl Med Technol 2007; 35:237-241

DOI: $10.2967 / j n m t .107 .040402$

\footnotetext{
Received Feb. 2, 2007; revision accepted May 7, 2007.

For correspondence or reprints contact: Arianna Massaro, CNMT, Nuclear Medicine Service, PET Unit, "S. Maria della Misericordia Rovigo" Hospital, Istituto Oncologico Veneto (IOV)-IRCCS, Viale Tre Martiri, 140, 45100, Rovigo, Italy.

E-mail: rubello.domenico@azisanrovigo.it

COPYRIGHT @ 2007 by the Society of Nuclear Medicine, Inc.
}

$\mathbf{I}$ n recent years, SPECT has gained an important role in scintigraphic evaluations because it is accurate and allows precise localization of the depth of radioactive lesions. At present, SPECT studies using radiotracers labeled with ${ }^{99 \mathrm{~m} T c-p e r t e c h n e t a t e}$ are well standardized, mainly because the radioisotope is characterized by optimal energy and high-statistic count rates for acquisitions with currently available $\gamma$-cameras $(1,2)$. In contrast, the choice of reconstruction parameters for medium-energy radioisotopes such as ${ }^{111}$ In is more difficult, mainly because they are characterized by a relatively low-statistic count rate $(3,4)$.

This study, which was based on a large series of consecutive patients imaged by ${ }^{111} \mathrm{In}$-pentetreotide SPECT for a neuroendocrine tumor, evaluated variability in reconstruction parameters in relation to patient body weight and the body region imaged, looking for the possibility of standardizing such parameters.

\section{MATERIALS AND METHODS}

At our center from January 2005 to December 2006, 124 consecutive patients affected by a clinically and biochemically proven neuroendocrine tumor were enrolled in the study. All patients were injected with $140-150 \mathrm{MBq}$ of ${ }^{111}$ In-pentetreotide at least $1 \mathrm{wk}$ after somatostatin analogs had been discontinued and underwent 4- and 24-h whole-body and spot planar imaging. Moreover, a 24-h SPECT acquisition of the head, chest, and abdomen was also systematically obtained.

The SPECT images were acquired on a dual-head $\gamma$-camera (E-CAM; Siemens) using medium-energy collimators, a step-andshoot acquisition protocol with no circular orbit, a $64 \times 64$ matrix, and $30 \mathrm{~s}$ per view for a total of 64 views. Two different reconstruction procedures were compared: first, an iterative method using ordered-subset expectation maximization (OSEM) with 10 iterations, like the method used in some previous studies on ${ }^{99 m} \mathrm{~m}$ Tc-technetium-labeled compounds (5); and second, filtered backprojection using a conventional technique previously described (6), with application and comparison of a variety of commercially available filters. 
TABLE 1

${ }^{111}$ In-Pentetreotide SPECT Reconstruction Parameters in Relation to Body Weight and Body Region

\begin{tabular}{lccccc}
\hline & \multicolumn{2}{c}{$\begin{array}{c}\text { Body weight }<70 \\
\mathrm{~kg}\end{array}$} & & \multicolumn{2}{c}{$\begin{array}{c}\text { Body weight } \geq 70 \\
\mathrm{~kg}\end{array}$} \\
\cline { 2 - 3 } \cline { 5 - 6 } Body region & Cutoff & Order & & Cutoff & Order \\
\hline Head & $0.30-0.40$ & $7-8$ & & $0.30-0.40$ & $7-8$ \\
Thorax & $0.35-0.50$ & $7-9$ & & $0.35-0.40$ & $7-9$ \\
Abdomen & $0.50-0.70$ & $7-9$ & & $0.55-0.80$ & $7-10$ \\
\hline
\end{tabular}

Scintigraphic images were examined in a masked fashion by 2 skilled nuclear medicine physicians. In cases of discrepancy, the diagnosis was reached by consensus.

Among the different reconstruction protocols used, we found the Butterworth filter to be most satisfactory in regard to spatial
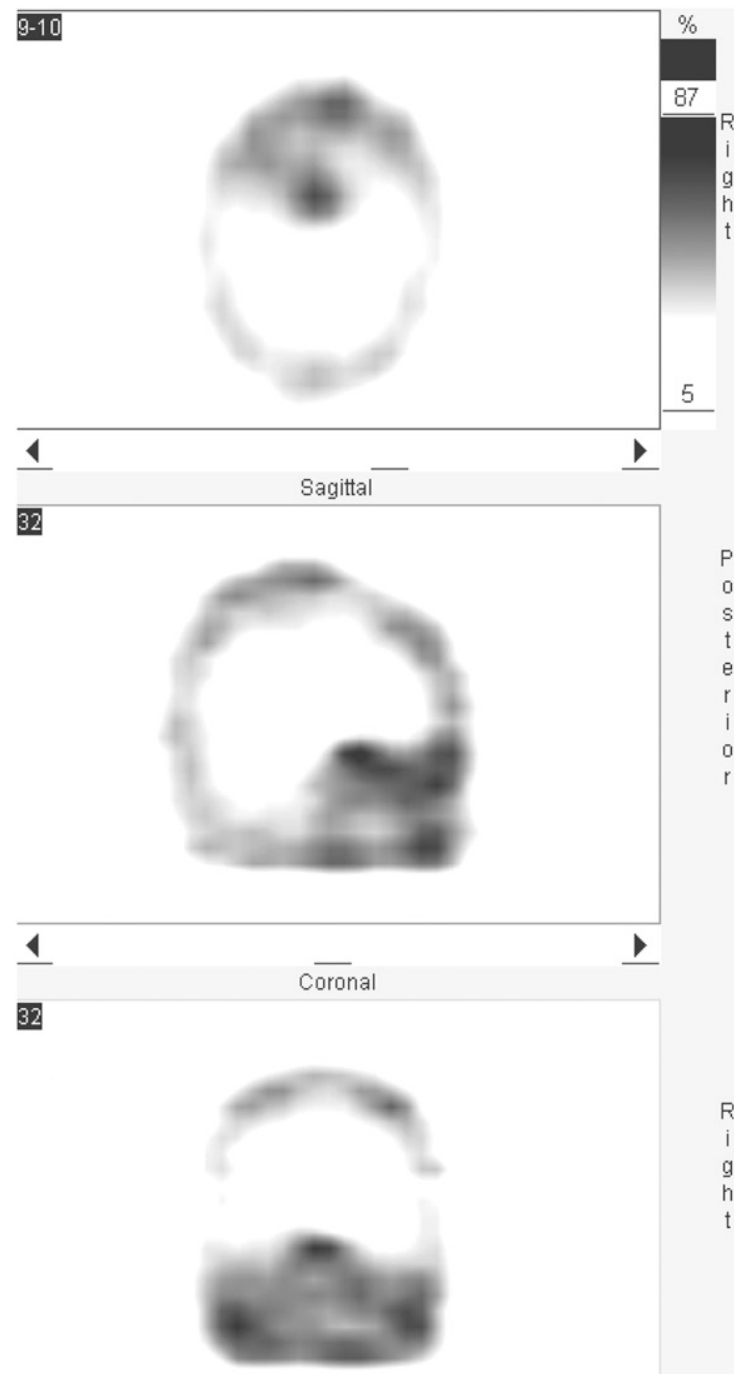

\section{4}

resolution, contrast, and image definition, producing a global improvement in image quality. Different orders and cutoffs were applied using this filter.

The patients were divided into 2 groups according to body weight (49 patients weighed more than $70 \mathrm{~kg} ; 65$ weighed $70 \mathrm{~kg}$ or less), and the SPECT images of the 3 body regions (head, chest, and abdomen) of each patient were compared.

\section{RESULTS}

Table 1 summarizes our results, and Figures 1-3 show examples of head, chest, and abdominal images obtained using the iterative and filtered backprojection methods. Image shape seemed slightly smoother with the iterative method, whereas the global quality of SPECT images was better defined with filtered backprojection with the Butterworth filter.

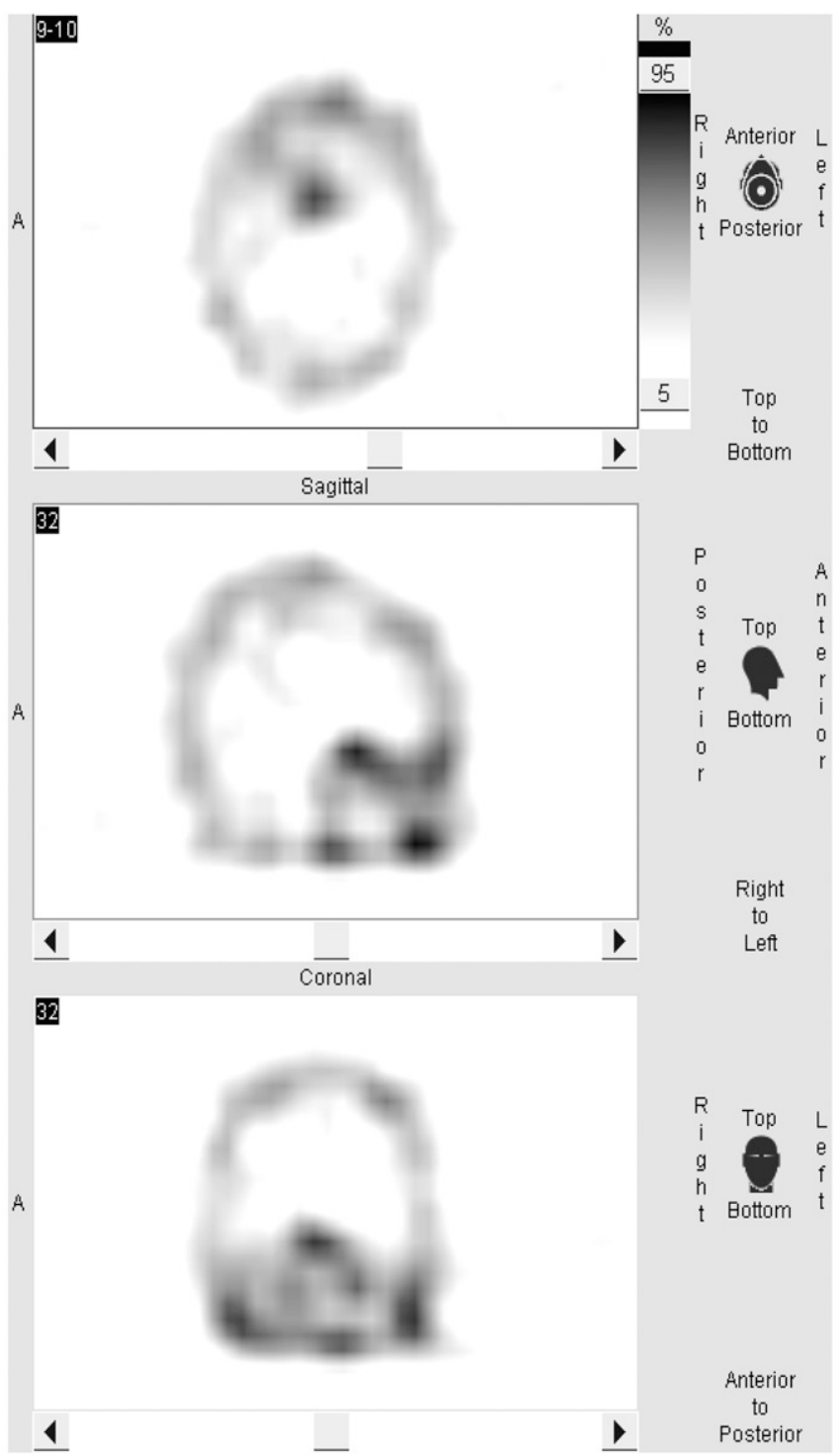

FIGURE 1. SPECT of head in patient with pituitary adenoma. Body weight was $74 \mathrm{~kg}$. (Left) Reconstruction by OSEM iterative method (from top to bottom: axial, sagittal, and coronal views). (Right) Reconstruction by filtered backprojection using Butterworth filter, with cutoff of 0.35 and order of 8 . 


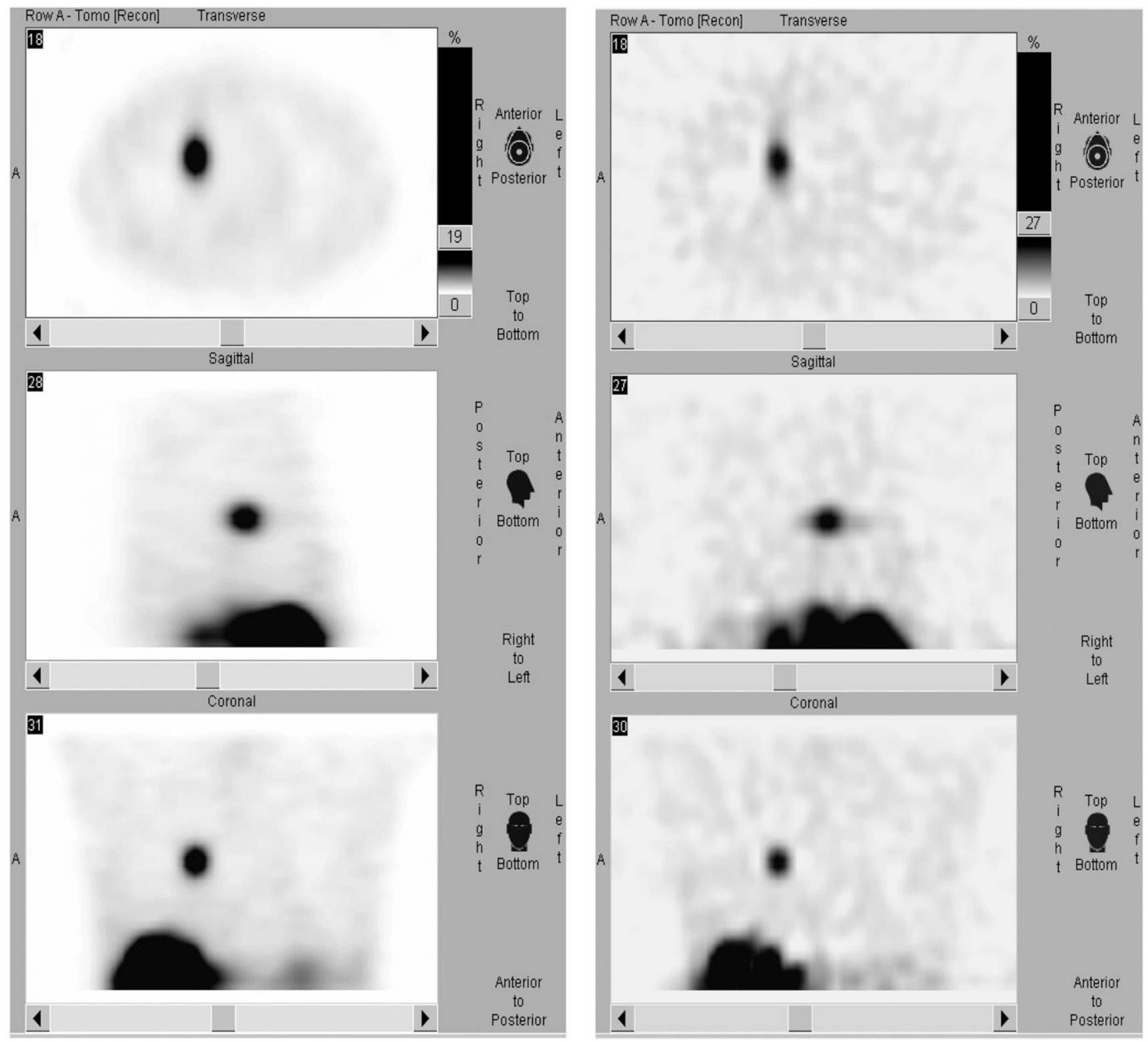

FIGURE 2. SPECT of chest in patient with typical bronchial carcinoid. Body weight was $68 \mathrm{~kg}$. (Left) Reconstruction by OSEM iterative method (from top to bottom: axial, sagittal, and coronal views). (Right) Reconstruction by filtered backprojection using Butterworth filter, with cutoff of 0.40 and order of 9 .

\section{DISCUSSION}

Our analysis of a large group of homogeneously studied patients showed that filtered backprojection using a Butterworth filter produced images of better resolution than did the iterative method. With the Butterworth filter, the reconstruction parameters were easily standardized for the head and chest but were variable for the abdomen, especially in patients weighing more than $70 \mathrm{~kg}$. This finding is explained by the presence of organs and tissues in the abdomen (such as the liver, spleen, bowel, and kidneys) characterized by moderate to high physiologic ${ }^{111} \mathrm{In}$-pentetreotide uptake $(3,4)$. Some investigators believe that a late, $72-\mathrm{h}$,
SPECT acquisition may partly overcome these interfering artifacts (7).

Regarding our finding that filtered backprojection using a Butterworth filter produced better images than did the OSEM iterative method, it has been reported that, for SPECT images with low count rates, the iterative method may ameliorate image quality (especially the shape of the lesion), providing smoother images (8). On the other hand, we obtained more defined SPECT images through filtered backprojection using a Butterworth filter, as might be explained by our injecting a relatively higher dose of ${ }^{111} \mathrm{In}$ pentetreotide - up to $150 \mathrm{MBq}$ per patient-than has been 


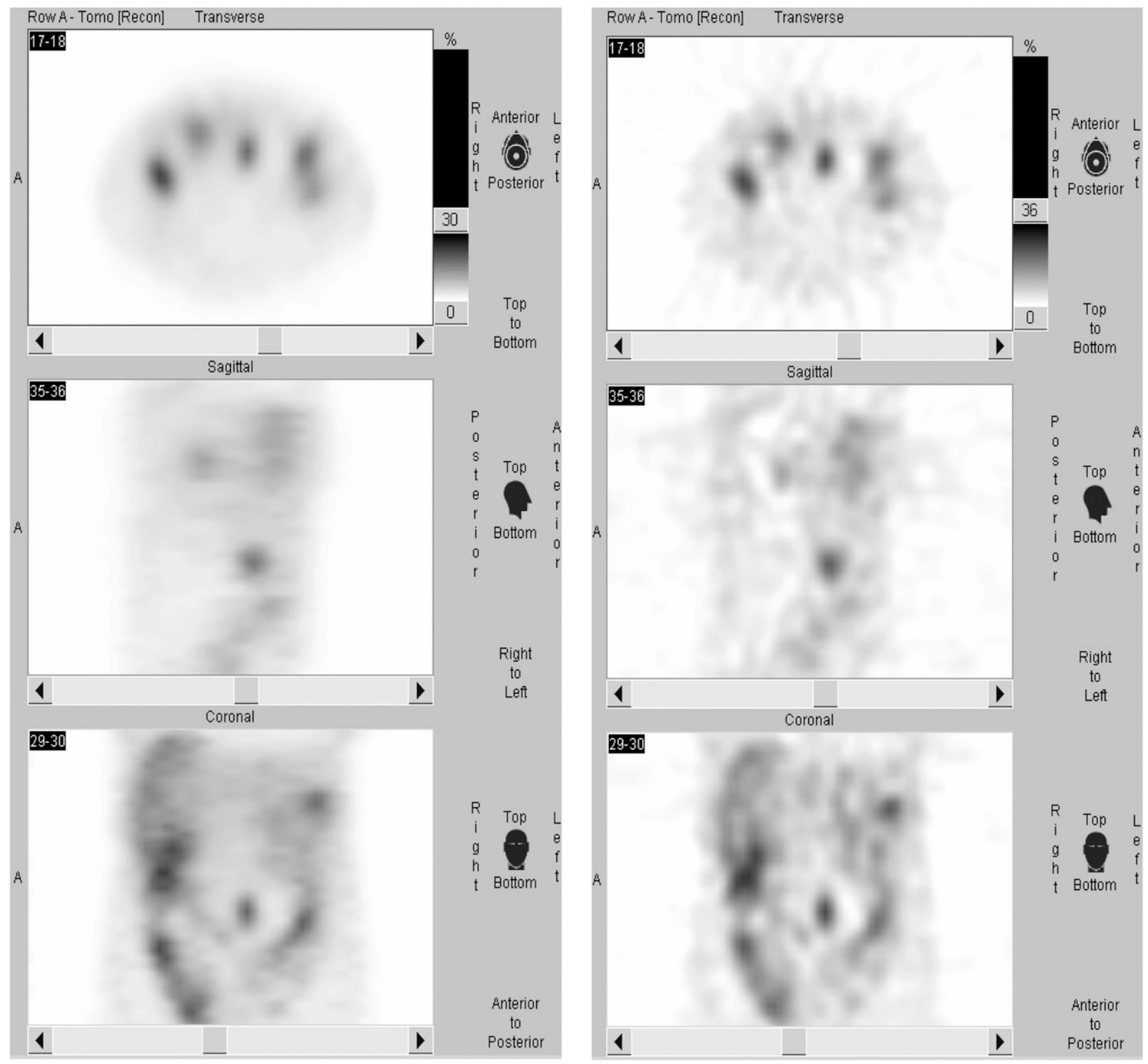

FIGURE 3. SPECT of abdomen in patient with ileal VIPoma (left periumbilical region). Body weight was $72 \mathrm{~kg}$. (Left) Reconstruction by OSEM iterative method (from top to bottom: axial, sagittal, and coronal views). (Right) Reconstruction by filtered backprojection using Butterworth filter, with cutoff of 0.65 and order of 9 .

used in other reported studies. Of course, a higher radiotracer dose is likely to produce a higher counting rate.

Thus, our data show that, in clinical practice, filtered backprojection using a Butterworth filter appears to be a good image-processing method for multiple (head, chest, and abdomen) ${ }^{111}$ In-pentetreotide SPECT examinations in the same patient.

Reconstruction parameters, although easily standardized for head and chest images, are not so easily standardized for images of the abdomen, where there are many timerelated interfering factors. Thus, in the case of abdominal images, reconstruction parameters can vary widely and depend on the skill of the operator.

\section{CONCLUSION}

Filtered backprojection using a Butterworth filter appears adequate for standardizing reconstruction parameters for ${ }^{111}$ In-pentetreotide SPECT of the head and chest. Processing of abdominal images is more operator-dependent. For a triple-SPECT examination (head, chest, and abdomen) in the same patient, administration of a $150-\mathrm{MBq}$ dose of ${ }^{111}$ In-pentetreotide is recommended.

\section{REFERENCES}

1. Morano GN, Sebyl JP. Technical overview of brain SPECT imaging: improving acquisition and processing of data. J Nucl Med Technol. 2003;31:191-195. 
2. Lin GS, Hines HH, Grant G, Taylor K, Ryals C. Automated quantification of myocardial ischemia and wall motion defects by use of cardiac SPECT polar mapping and 4-dimensional surface rendering. J Nucl Med Technol. 2006;34: 3-17.

3. De Jong M, Valkema R, van Gameren A, et al. Inhomogeneous localization of radioactivity in the human kidney after injection of [(111)In-DTPA]octreotide. J Nucl Med. 2004;45:1168-1171.

4. Roach PJ, Schembri GP, Ho Shon IA, Bailey EA, Bailey DL. SPECT/CT imaging using a spiral CT scanner for anatomical localization: impact on diagnostic accuracy and reporter confidence in clinical practice. Nucl Med Commun. 2006;27: 977-987.
5. Zakavi SR, Zonoozi A, Kakhki VD, Hajizadeh M, Momennezhad M, Ariana K. Image reconstruction using filtered back projection and iterative method: effect on motion artifacts in myocardial perfusion SPECT. J Nucl Med Technol. 2006;34: 220-223.

6. Leong LK, Kruger RL, O'Connor MK. A comparison of the uniformity requirements for SPECT image reconstruction using FBP and OSEM techniques. J Nucl Med Technol. 2001;29:79-83.

7. Rufini V, Calcagni ML, Baum RP. Imaging of neuroendocrine tumors. Semin Nucl Med. 2006;36:228-247.

8. Pan X. A general approach for multidimensional smoothing. Med Phys. 1998; 25:562-570. 